\title{
School-Based Nutrition Education Intervention Improves Nutrition Knowledge and Lipid Profile among Overweight/Obese Children
}

\author{
Phyllis Addo ${ }^{1}$, Eric Adua ${ }^{2}$, Obed Akwaa Harrison ${ }^{1}$, Rose Otema Baah ${ }^{1}$, \\ Alex Kojo Anderson ${ }^{3} \&$ Matilda Steiner-Aseidu ${ }^{1}$ \\ ${ }^{1}$ Department of Nutrition and Food Science, University of Ghana \\ ${ }^{2}$ School of Medical and Health Sciences, Edith Cowan University, Australia \\ ${ }^{3}$ Department of Foods and Nutrition, University of Georgia, U.S.A \\ Correspondence: Matilda Steiner-Asiedu, Department of Nutrition and Food Science P. O. Box LG 134 Legon - \\ Accra, Ghana. Tel: 233-541-260-704. E-mail: tillysteiner@gmail.com
}

Received: May 4, 2017 Accepted: June 3, 2017 Online Published: September 6, 2017

doi:10.5539/gjhs.v9n10p109 URL: https://doi.org/10.5539/gjhs.v9n10p109

\begin{abstract}
Many children in Ghana do not meet the dietary and physical activity recommendations for their health due to several reasons including limited nutrition education intervention (NEI) programmes. NEI provides children with information on knowledge, attitudes and practices (KAPs) required to ensure proper dietary intake and physical activity. In this intervention study, we recruited eighty (80) overweight and obese students aged 11-15 years from two schools in the Ga-East municipality of Ghana. Anthropometric, biochemical, dietary and physical activity information were collected on the two groups before and after three months of NEI. Between group comparisons (test and control); before and after interventions were performed using student $t$-tests. It was shown that NEI improved nutrition knowledge (mean change $=5.13, \mathrm{p}<0.01$ ), attitude (mean change $=2.75, \mathrm{p}<0.01$ ) but not practice (mean change $=-1.42, \mathrm{p}<0.05$ ) in overweight and obese children. Although anthropometric indicators did not improve with NEI, serum lipid profile of participants improved as indicated by the following mean changes: TC [-1.22, 95\%CI (-1.90 -0.55)] mg/dL, HDL-c [-0.19, 95\% CI $(-0.38,0.00)] \mathrm{mg} / \mathrm{dL}$, LDL-c [-0.90, 95\%CI (-1.52, $-0.28)] \mathrm{mg} / \mathrm{dL}$ and TG $[(-0.66,95 \% \mathrm{CI}(-1.23,-0.09)] \mathrm{mg} / \mathrm{dL}$. Our findings show that NEI undertaken within a relatively short period of time could have positive effects on lipid profile, knowledge and attitudes of school children, and in turn, promote the fight against childhood obesity, and improve the health and wellbeing of children.
\end{abstract}

Keywords: obesity, overweight, lipid profile, nutrition education intervention

\section{Introduction}

Globally, one in every ten school-aged children and adolescents are overweight or obese; a total of 155 million. Of this number, 30-45 million are obese; which constitutes 2-3\% of the world's children aged 5-17 years (Lobstein et al., 2004). Many of these children and adolescents live in low-to middle income countries like Ghana. According to a 2008 report from the Global School-based Health Survey, 6.6\% and 1.2\% of Ghanaian adolescents aged 13-15 years were overweight and obese respectively (Global School-based Health Survey, 2008) and this has been predicted to increase in the near future. The increasing prevalence of overweight and obesity among these young people is mainly due to increased consumption of highly processed and fried foods, high calorie foods, with insufficient or no intake of fruits and vegetables. Among the estimated $72 \%$ of students who consume fruits and vegetables at least once a day, only 20\% meet the recommended servings (Global School-based Health Survey, 2008). Alongside these factors, obesity is triggered by physical inactivity. Reports from the Regenerative Health and Nutrition Programme in Ghana show that two out of every three children have insufficient physical activity (Regenerative Health and Nutrition Programme, 2007).

Over the years, several school-based nutrition and health promotion programs have been implemented in Ghana. These include the national school feeding program and the micronutrient supplementation program (A. Alhassan \& F. Alhassan, 2014; Atta \& Manu, 2015). Although these programs to a lesser extent have met the nutritional needs of majority of children, they mainly focused on addressing the prevailing under-nutrition problems rather than obesity, hence the need for a nutrition education intervention (NEI) that will provide information on the 
knowledge, attitudes and practices (KAPs) needed to inform the right decision making on dietary and physical activity choices. Therefore, this study examines the effect of school-based NEI on KAPs and nutritional status among overweight and obese children in Ghana.

\section{Methods}

\subsection{Study Design}

A prospective cohort study design was employed. We recruited 80 overweight/obese students aged 11-15 years from an ongoing childhood obesity study. However, analysis was done using completed data from 54 participants (24 in the test group and 30 in wait-list control group). This intervention study used a purposive sampling approach to select 2 schools out of the 32 schools that participated in the baseline cross-sectional study (Figure 1). These schools were selected because of the similar characteristics they shared at baseline. Detailed description of the lifestyle and nutrition profile of participants at baseline has been published elsewhere (Steiner-Asiedu et al., 2012). Selection of participants was done using the 2007 WHO sex specific BMI-for-age charts. The two groups were randomly assigned to intervention group and control group by coin tossing.

Study participants were followed throughout the school year (2011-2012). At the beginning and ending of the NEI, data were gathered on the anthropometric and biochemical profile of participants as well as their KAPs on nutrition and physical activities. The NEI comprised a class of nutritional recommendations once every week, administration of educational fliers and a pedometer to monitor physical activities.

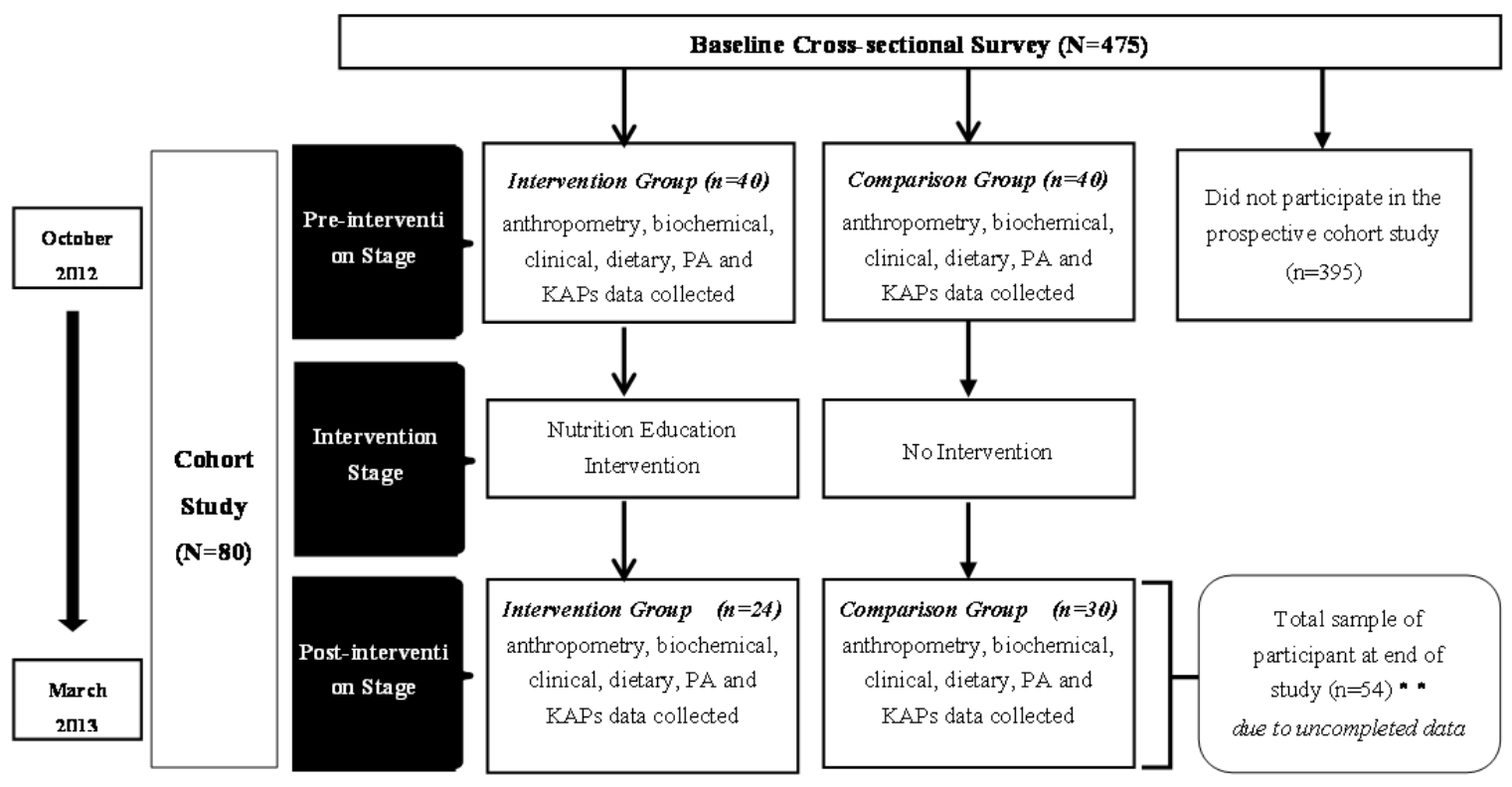

Figure 1. Overview of study methodology

\subsection{Description of Intervention}

The NEI provided participants with knowledge and practical skills required to attain healthy dietary and physical activity behaviours. Also, posters with nutrition information were set up and fliers given to participants to reinforce and enhance further understanding of the lessons. Newsletters were also sent to parents or guardians of participants to educate them on how to help their wards practice healthy lifestyles. The wait-list control group (WLCG) did not receive any of such information during the six weeks of the intervention.

\subsection{Measurements}

\subsubsection{Sociodemographic Data}

A validated semi-structured questionnaire was used to obtain information on socio-demographics including age, gender and ethnicity. Also data on the educational level and occupational status of parents was collected.

\subsubsection{Anthropometric Data}

Weight in kilograms $(\mathrm{kg})$ was measured on calibrated weighing scale (Model QIE-2003A; EMC China). Height in centimeters $(\mathrm{cm})$ was measured on a calibrated stadiometer (Model HM200P; Charder USA) following standard 
protocol. Height and weight were measured twice, rounded to the nearest $0.1 \mathrm{~cm}$ and $0.1 \mathrm{~kg}$, respectively, and the average of the two measurements was used to calculate the body mass index (BMI). Participants were classified as overweight (BMI z score between 1 SD and 2 SD) or obese (BMI z score $>2$ SD) according to the 2007 World Health Organization (WHO) sex specific BMI-for-age charts (World Health Organisation, 2007). Waist and hip circumferences $(\mathrm{cm})$ were measured twice with a flexible non-stretch tape measure while the subjects were in a relaxed standing position. Waist circumference was measured at the umbilical level and hip circumference was measured at the maximum protuberance of the buttocks. The means of these two measurements were used to calculate the waist- to- hip ratio (WHR). The percentage body fat of each participant was also measured via bioelectrical impedance analysis (BIA) using the hand-held body fat analyzer (Model HBF360; Omron Japan). Measurements were taken for each participant after an overnight fast at similar times of the day at pre- and post-assessment. The conventional cut-off ( $\geq 25 \%$ for boys and $\geq 30 \%$ for girls) defined by Williams et al., (1992) was used.

\subsubsection{Biochemical and Clinical Data}

Serum lipid profiles including total cholesterol (TC), high density lipoprotein cholesterol (HDL-c), triglycerides (TG) and low density lipoprotein cholesterol (LDL-c) were examined using the Lipid Profile Analyzer (Model CardiChek PA; POCD Australia) while fasting blood glucose (FBG) and haemoglobin (Hb) concentration were determined using the HemoCue Glucose Analyzer(Model $201^{+}$; HemoCue Sweden) and the Hb analyzer (Model $201^{+}$; HemoCue Sweden) respectively. Blood samples were taken in the morning after an overnight fast. Using cut-offs for children by the American Heart Association, acceptable TC was defined as $<170 \mathrm{mg} / \mathrm{dL}$, borderline as $(170-199) \mathrm{mg} / \mathrm{dL}$ and high TC as $\geq 200 \mathrm{mg} / \mathrm{dL}$. Acceptable FBG levels were defined as $<90 \mathrm{mg} / \mathrm{dL}$ according to the cut-offs by National Diabetes Education Program (2008). Systolic blood pressure (SBP) and diastolic blood pressure (DBP) were measured with an automatic sphygmomanometer (Model HEM-712CN2; Omron Japan). Blood pressure was measured twice on the left arm of participants in a seated relaxed position at least 2-minutes intervals and the average of the two measurements was used. These were recorded to the nearest $2 \mathrm{mmHg}$.

\subsubsection{KAPs Data}

A structured questionnaire adapted from the Healthy Lifestyle in Children (HELIC) study (SitiSabariah et al., 2006) was used to measure nutrition knowledge, attitude and practices. Participants completed a 50-item multiple-choice or true/false questionnaire before and after intervention. Responses were scored and correct answers were summed up to yield a total score. There were 30 questions on the nutrition knowledge item which had either a four answer option or true/false option. Correct and incorrect responses on the nutrition knowledge were allocated 2 and 0 point respectively. Thus, a highest score in this section was 60 . There were 10 questions each for the attitude and practice items. The attitude item was on a 3 point-scale while the practice item was assessed on a 4 point-scale, ranging from 'almost every day' to 'never'. Favourable and unfavourable options were given 2 and 0 points, respectively and an intermediate option (neutral) was allocated 1 point. The respective maximum and minimum scores for these sections were 20 and 0 respectively. The overall highest score was 100 and lowest score 0 . Total scores for the KAPs items were then ranked and classified in 4 different levels; weak $\left(<25^{\text {th }}\right.$ percentile), moderate (between $25^{\text {th }}$ and $50^{\text {th }}$ percentile), good (between $50^{\text {th }}$ and $75^{\text {th }}$ percentile) and excellent (above $75^{\text {th }}$ percentile).

\subsection{Statistical Analysis}

All data analyses were conducted using the Statistical Package for Social Sciences (SPSS version 16.0). Differences in continuous variables (before and after intervention) including age, body weights and BMI were analyzed using the student $t$-tests. These data were presented as mean \pm standard deviation. Categorical variables like gender, age group, ethnicity and educational levels were analyzed using Chi-square tests and were presented as frequencies, percentages or proportions. For all comparisons, p-value $<0.05$ was considered statistically significant.

\subsection{Ethical Consideration}

Ethical clearance was obtained from the Institutional Review Board of the Noguchi Memorial Institute for Medical Research, University of Ghana, after the research protocol had been reviewed fully. Approval was also obtained from school authorities in all the schools where the study was carried out. Written informed consent was obtained from parents/guardian of each participant.

\section{Results}

Of the 80 participants recruited into this study, 26 were excluded from the analyses due to incomplete data. The remaining 54 participants were categorized into two; intervention group $(n=24)$ and the comparison group $(n=30)$. These two groups were comparable in gender $\left(\chi^{2}=0.841, p=0.359\right)$, age $(t=1.086, p=0.283)$ and other indices like 
pupil's class standing and parental occupation (Table 1).

The changes in the anthropometric indicators (weight, BMI, WC, WHR and percent body fat) assessed were all not significantly different between children who received the school-based nutrition education intervention and those in the control group (Table 2). This indicates that body composition did not improve with nutrition education intervention. But within the groups, changes were observed in certain variables before and after the intervention. In the intervention group for instance the WC and WHR reduced from $86.19 \mathrm{~cm}$ to $85.22 \mathrm{~cm}$ and 0.85 to 0.84 respectively.

The pre- and post-test mean values for the biochemical and clinical indicators of the intervention and comparison groups are presented in Table 3 below. At baseline, apart from the mean $\mathrm{Hb}$ values, there was no significant difference in the mean values for all the other variables being compared between the intervention and comparison groups. In comparing the lipid profile between the two groups, it was observed that, there were significant improvements in the post intervention mean values for the intervention group as indicated by the mean change in total cholesterol (Mean change $=-1.22, \mathrm{p}<0.01$ ), HDL (Mean change $=-0.19, \mathrm{p}<0.05$ ), LDL (Mean change=-0.90, $\mathrm{p}<0.05$ ) and triglycerides (Mean change $=-0.66, \mathrm{p}<0.05$ ). Within the intervention group, both biological and clinical indicators improved, with statistical significance in the mean triglyceride value (15.44 to 10.95$) \mathrm{mg} / \mathrm{dL}$ and diastolic blood pressure (70.46 to 67.19) $\mathrm{mmHg}$.

Responses on nutrition knowledge were ranked and classified as weak, moderate, good and excellent based on percentiles. Generally, there was improvement in knowledge. At post-test the percentage of participants who had below moderate performance (i.e. fell below $50^{\text {th }}$ percentile) reduced from $45.8 \%$ to $41.7 \%$ whilst those who had good or excellent scores (i.e. from $50^{\text {th }}$ percentile and above) increased from $54.2 \%$ to $58.3 \%$. Nonetheless, in the individual groups, certain variations occurred, for instance, in the weak and excellent groups there was a reduction in numbers at post-test whereas the moderate and good categories experienced an increase (Figure 2).

Table 1. Age and anthropometric variables of participants

\begin{tabular}{llll}
\hline Variable & Total $(\mathrm{n}=54)$ & Intervention $(\mathrm{n}=24)$ & Control $(\mathrm{n}=30)$ \\
\hline Age (years) & $13 \pm 1.0$ & $12 \pm 1.0$ & $13.0 \pm 1.0$ \\
BMI kg/m ${ }^{2}$ & $26.8 \pm 3.3$ & $27.2 \pm 3.9$ & $26.4 \pm 2.7$ \\
Body fat $(\%)$ & $31.9 \pm 5.4$ & $31.8 \pm 6.0$ & $32.1 \pm 5.0$ \\
WHR & $0.8 \pm 0.1$ & $0.9 \pm 0.0$ & $0.8 \pm 0.1$ \\
WC $(\mathrm{cm})$ & $85.3 \pm 5.1$ & $86.2 \pm 4.9$ & $84.3 \pm 5.2$ \\
\hline
\end{tabular}


Table 2. Mean differences in anthropometric indicators between test and control groups

\begin{tabular}{|c|c|c|c|}
\hline \multirow[t]{2}{*}{ Factor } & Intervention & Comparison & Mean Difference $\dagger$ \\
\hline & Mean $(95 \% \mathrm{CI})$ & Mean $(95 \% \mathrm{CI})$ & $(95 \% \mathrm{CI})$ \\
\hline \multicolumn{4}{|l|}{ Weight, kg } \\
\hline Pre-test & $68.35(63.52,73.17)$ & $64.36(60.86,67.85)$ & $3.99(-1.68,9.67)$ \\
\hline Post-test & $69.48(64.74,74.21)$ & $65.33(61.72,68.95)$ & $4.14(-1.57,9.85)$ \\
\hline Mean Difference $\dagger \dagger$ (95\% CI) & $-1.13(-2.21,-0.04)^{\mathrm{a}}$ & $-0.98(-2.27, \quad 0.32)$ & \\
\hline \multicolumn{4}{|l|}{$\mathrm{BMI}, \mathrm{kg} / \mathrm{m}^{2}$} \\
\hline Pre-test & $27.23(25.59,28.87)$ & $26.44(25.44,27.45)$ & $0.79(-1.01,2.59)$ \\
\hline Post-test & $27.27(25.69,28.85)$ & $26.32(25.34,27.30)$ & $0.95(-0.79,2.69)$ \\
\hline Mean Difference $\dagger \dagger(95 \% \mathrm{CI})$ & $-0.04(-0.97,0.89)$ & $0.12(-0.26,0.50)$ & \\
\hline \multicolumn{4}{|l|}{$\mathrm{WC}, \mathrm{cm}$} \\
\hline Pre-test & $86.19(83.75,88.63)$ & $84.30(81.68,86.91)$ & $1.90(-1.67,5.46)$ \\
\hline Post-test & $85.22(82.25,88.20)$ & $84.04(81.37,86.70)$ & $1.19(-2.72,5.09)$ \\
\hline Mean Difference $\dagger \dagger(95 \%$ CI) & $0.97(0.11, \quad 1.82)^{\mathrm{a}}$ & $0.26(-0.88, \quad 1.40)$ & \\
\hline \multicolumn{4}{|l|}{ WHR } \\
\hline Pre-test & $0.85(0.84,0.87)$ & $0.84(0.81,0.86)$ & $0.01(-0.02,0.05)$ \\
\hline Post-test & $0.84(0.82,0.86)$ & $0.81(0.79,0.84)$ & $0.03(0.00,0.06)$ \\
\hline Mean Difference $\dagger \dagger(95 \%$ CI) & $0.01(0.00, \quad 0.02)^{\mathrm{a}}$ & 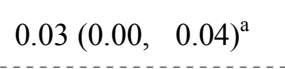 & \\
\hline \multicolumn{4}{|l|}{ Body Fat, \% } \\
\hline Pre-test & $31.77(29.27,34.27)$ & $32.05(30.20,33.91)$ & $-0.28(-3.25,2.69)$ \\
\hline Post-test & $31.47(28.84,34.10)$ & $31.04(29.44,32.64)$ & $0.43(-2.45,3.30)$ \\
\hline Mean Difference $\dagger \dagger$ (95\% CI) & $0.30(-1.05, \quad 1.65)$ & $1.01(0.19,1.83)^{\mathrm{a}}$ & \\
\hline
\end{tabular}

BMI indicates body mass index; WC, waist circumference and WHR, waist-to-hip ratio;

a Significantly different between pre-test and post-test within group $(\mathrm{p}<0.05)$;

$†$ Calculated from mean values (intervention mean - comparison mean);

$\dagger \dagger$ Calculated from test scores values (pre-test mean - post-test mean); 
Table 3. Mean differences in biochemical and clinical indicators between test and control groups

\begin{tabular}{|c|c|c|c|}
\hline \multirow[t]{2}{*}{ Factor } & Test Group & Control Group & Mean Difference $\dagger$ \\
\hline & Mean $(95 \% \mathrm{CI})$ & Mean $(95 \% \mathrm{CI})$ & $(95 \% \mathrm{CI})$ \\
\hline \multicolumn{4}{|l|}{$\mathrm{FBG}, \mathrm{mg} / \mathrm{dl}$} \\
\hline Pre-test & $110.17(103.03,117.32)$ & $125.14(113.79,136.50)$ & $-11.08(-26.63,4.48)$ \\
\hline Post-test & $109.57(102.98,116.69)$ & $118.89(107.42,130.36)$ & $-16.05(-32.58,-0.48)$ \\
\hline Mean Difference $\dagger \dagger(95 \%$ CI) & $0.60(-1.07,2.29)$ & $1.23(-1.12,25.81)$ & \\
\hline \multicolumn{4}{|l|}{$\mathrm{Hb}, \mathrm{mg} / \mathrm{dl}$} \\
\hline Pre-test & $12.83(12.42,13.23)$ & $11.40(10.58,12.23)$ & $1.42(0.45,0.40)^{\mathrm{b}}$ \\
\hline Post-test & $12.50(11.98,13.01)$ & $12.29(11.90,12.68)$ & $0.21(-0.41,0.83)$ \\
\hline Mean Difference $\uparrow \dagger(95 \%$ CI) & $0.33(-0.21,0.87)$ & $-0.89(-1.56,-0.21)^{\mathrm{a}}$ & \\
\hline \multicolumn{4}{|l|}{$\mathrm{TC}, \mathrm{mg} / \mathrm{dl}$} \\
\hline Pre-test & $66.05(53.67,78.42)$ & $77.47(66.48,88.46)$ & $-0.63(-1.53,0.26)$ \\
\hline Post-test & $57.14(52.41,61.86)$ & $79.12(68.72,89.51)$ & $-1.22(-1.90,-0.55)^{\mathrm{b} * *}$ \\
\hline Mean Difference $\dagger \dagger(95 \%$ CI) & $0.50(-0.18,1.17)$ & $-0.09(-0.33,0.14)$ & \\
\hline \multicolumn{4}{|l|}{$\mathrm{HDL}, \mathrm{mg} / \mathrm{dl}$} \\
\hline Pre-test & $18.79(16.98,20.59)$ & $21.70(19.14,24.26)$ & $-0.16(-0.34,0.02)$ \\
\hline Post-test & $17.69(15.31,20.08)$ & $21.13(18.72,23.54)$ & $-0.19(-0.38,0.00)^{b}$ \\
\hline Mean Difference $\uparrow \dagger(95 \%$ CI) & $0.60(-0.07,0.19)$ & $0.03(-0.05,1.11)$ & \\
\hline \multicolumn{4}{|l|}{$\mathrm{LDL}, \mathrm{mg} / \mathrm{dl}$} \\
\hline Pre-test & $44.17(32.01,56.34)$ & $51.15(41.29,61.73)$ & $-0.41(-1.26,0.45)$ \\
\hline Post-test & $37.25(33.02,41.49)$ & $53.43(43.85,62.98)$ & $-0.90(-1.52,-0.28)^{\mathrm{b}}$ \\
\hline Mean Difference $\dagger \uparrow(95 \%$ CI $)$ & $0.38(-0.28,1.05)$ & $-0.11(-0.33,0.11)$ & \\
\hline \multicolumn{4}{|l|}{$\mathrm{TC} / \mathrm{HDL}$} \\
\hline Pre-test & $3.59(3.01,4.17)$ & $3.98(3.17,4.79)$ & $-0.39(-1.41,0.62)$ \\
\hline Post-test & $3.50(3.00,4.00)$ & $4.07(3.40,4.75)$ & $-0.57(-1.43,0.29)$ \\
\hline Mean Difference $\dagger \dagger(95 \%$ CI) & $0.09(-0.62,0.80)$ & $-0.09(-0.46,0.28)$ & \\
\hline \multicolumn{4}{|l|}{ Trig, mg/dl } \\
\hline Pre-test & $15.44(11.37,19.50)$ & $21.28(12.58,29.98)$ & $-0.32(-0.89,0.24)$ \\
\hline Post-test & $10.95(10.32,11.58)$ & $22.79(13.51,32.07)$ & $-0.66(-1.23,-0.09)^{b}$ \\
\hline Mean Difference $\dagger \dagger(95 \%$ CI) & $0.25(0.03,0.47)^{\mathrm{a}}$ & $-0.08(-0.30,0.13)$ & \\
\hline \multicolumn{4}{|l|}{$\mathrm{SBP}, \mathrm{mm} \mathrm{Hg}$} \\
\hline Pre-test & $118.29(113.35,123.23)$ & $115.52(112.30,118.73)$ & $2.78(-2.77,8.32)$ \\
\hline Post-test & $114.71(111.51,117.91)$ & $111.72(107.14,116.29)$ & $2.99(-2.75,8.73)$ \\
\hline Mean Difference $\dagger \dagger(95 \%$ CI) & $3.58(-1.60,8.76)$ & $3.80(-0.24,7.84)$ & \\
\hline \multicolumn{4}{|l|}{$\mathrm{DBP}, \mathrm{mm} \mathrm{Hg}$} \\
\hline Pre-test & $70.46(66.79,74.13)$ & $71.97(69.37,74.46)$ & $-1.46(-5.69,2.77)$ \\
\hline Post-test & $67.19(62.11,72.27)^{\mathrm{a}}$ & $70.62(68.34,72.89)$ & $-3.43(-8.48,1.62)$ \\
\hline Mean Difference $\dagger \dagger(95 \%$ CI) & $3.27(-1.25,7.80)$ & $1.30(-1.03,3.63)$ & \\
\hline
\end{tabular}

FBG indicates fasting blood glucose; Hb, hemoglobin; TC, total blood cholesterol, HDL, high-density lipoprotein; LDL, low-density lipoprotein; Trig, triglycerides; SBP, systolic blood pressure and DBP, diastolic blood pressure.

$\dagger$ Calculated from mean values (intervention mean - comparison mean) 
$\dagger$ Calculated from mean values (pre-test mean - post-test mean)

a Significantly different between pre-test and post-test within group $(\mathrm{p}<0.05)$

b Significantly different between test and control groups (independent t-test)

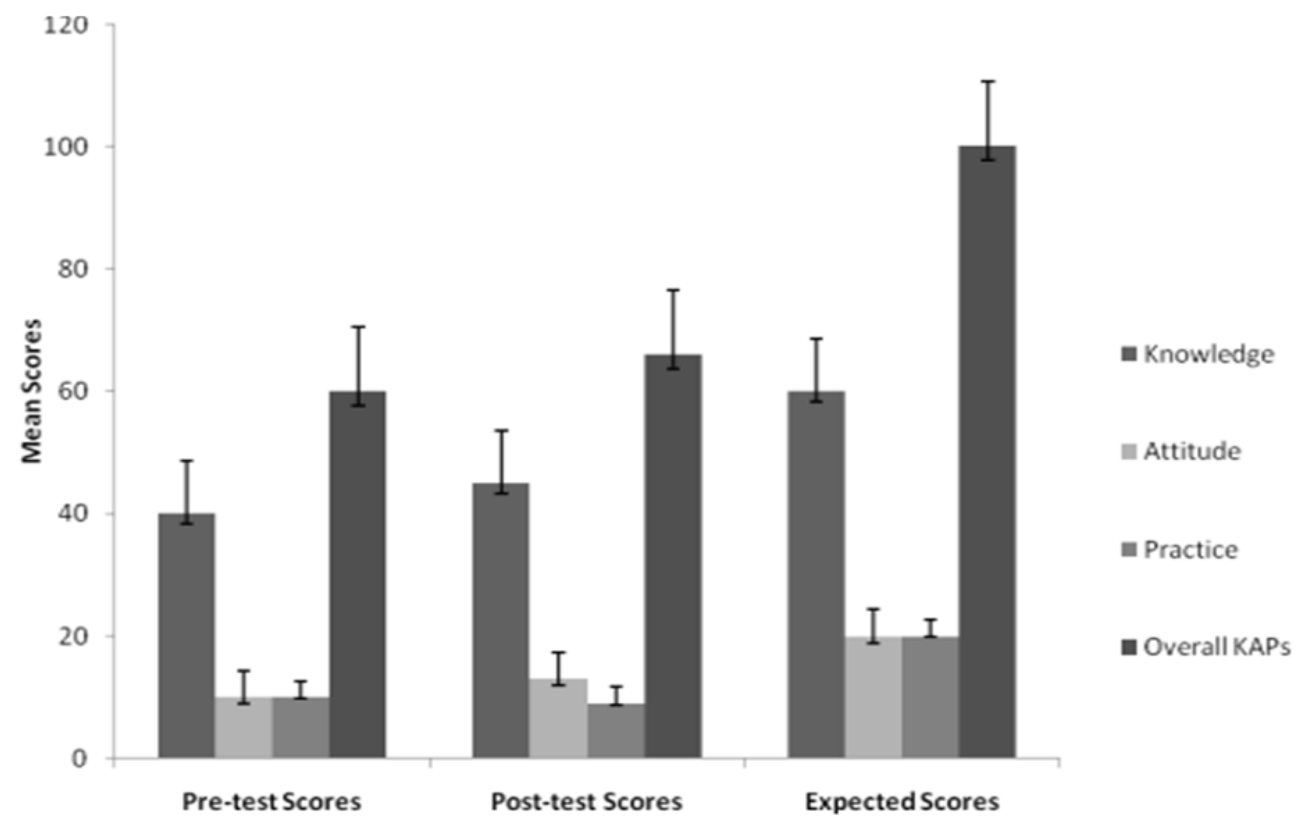

Figure 2. Mean scores of KAPs at pre- and post-test among the test group

\section{Discussion}

NEI is crucial to the attainment of healthy dietary and physical activity behaviour and subsequently betternutrition and health status of individuals. The results of our study showed that NEI produced significant improvements in nutrition knowledge (mean change $=5.13, \mathrm{p}=0.005$ ) and attitude (mean change $=2.75, \mathrm{p}=0.002$ ) but a decrease in practice (mean change $=-1.42, \mathrm{p}=0.026$ ) (Figure 2). The improvement in knowledge and attitude is consistent with other studies which showed that school-based nutrition education improved pre-schoolers' lifestyle behaviours resulting in beneficial changes in parents' attitudes to planning their children's diets and their own personal eating habits (Hu et al., 2010; Shariff et al., 2008).

The period of implementation for nutrition intervention is critical and has been correlated with behavioral outcomes. A study by Contento et al. showed that in order to observe sufficient improvement in knowledge and behavioral changes, intervention implementation should last for at least 50 hours (Contento et al., 1995). Therefore, the decrease in practice observed in the present study could be attributed to the short implementation period.

Factors like changes in the physical environment and accessibility to healthy foods are necessary to promoting positive behavioral and nutritional outcomes in children. This has been emphasized by researches that have shown that limited food availability and accessibility by children is a major setbackto achieving a healthy dietary habit regardless of adequate nutrition awareness, knowledge and positive attitude towards healthy nutrition (Kaiser \& Townsend, 2005; Sherman \& Muehlhoff, 2007).

There were generally no significant differences in the anthropometrics (weight, BMI, WC, WHR and percent body fat) between intervention and control groups following NEI (Table 2). However, there were positive changes observed within intervention group with the 'before and after' measurements. Notable among these are the average WC and WHR which reduced from $86.19 \mathrm{~cm}$ to $85.22 \mathrm{~cm}$ and 0.85 to 0.84 respectively. A possible reason for this could be the influence of the age group used in this study. The mean age of participants was $12 \pm 1.0$ years and this period has been identified by researchers as a time where puberty influences weight, height and other body composition; making anthropometric assessment of nutritional status very complicated (Bowman \& Russell, 2001). 
A study by Eliakem et al. (2002) reported a significant decrease in body weight and BMI following a 3 months dietary-behavioural-exercise intervention (Eliakim et al., 2002). Their results further showed that obese children who continued the intervention programme for 6 months maintained a decrease in BMI while those who did not participate gained weight and increased BMI. It could therefore be deduced from this study that the duration of an intervention of this sort may influence anthropometric variables. Our study assessed effects of the intervention only one month after intervention. The relatively short period may have contributed to the lack of significant differences in anthropometric measures because anthropometric indices, unlike biomarkers take relatively longer periods to manifest.

Intervention also improved the lipid profile of participants; TC (mean change $=-1.22, p=0.001$ ), HDL-c (mean change $=-0.19, \mathrm{p}=0.045)$, LDL-c (mean change $=-0.90, \mathrm{p}=0.005)$ and TG (mean change $=-0.66, \mathrm{p}=0.024)$ (Table 3). A previous study observed similar findings to that of the current study where NEI programmes improved lipid profile of children (Williams et al., 1992). These findings suggest that NEI implemented at relatively short duration ( 1 hour per week for 6 weeks) could have positive effects on the lipid profile, knowledge and attitude of school children.

The study employed several techniques to ensure the effectiveness of the intervention. Strategies like display of posters on school compound, distribution of fliers, provision of pedometers to help participants monitor their physical activities and sending newsletters containing nutritional information to parents were all done to raise awareness of healthy nutrition and lifestyle and to reinforce lessons taught to students during the intervention period. Nonetheless, it should be noted that there were some limitations in our study in terms of the implementation of the intervention and the evaluation instrument used. This may have influenced the study outcome. For instance, this intervention was carried out in a school where the environment was not controlled (in terms of food sold or served at the school). All indicators were also assessed a month after the intervention ended. Hence the short gap between completion and assessment time may have contributed to the observed improvement in the biochemical indicators. Furthermore, due to limited financial resources, the study was carried out in a private school in the urban area. Thus, the effectiveness of the intervention and results may not be generalizable to children in the public schools and rural areas with different socioeconomic backgrounds.

\section{Conclusion}

The need for the inclusion of the NEI in schools for children has been recognized. The present study shows that NEI has positive effects on biochemical profiles, knowledge and attitudes of school children which are essential to managing childhood obesity and its associated co-morbidities. However, tackling childhood overweight and obesity as whole will require a multidisciplinary team comprising dieticians, health service providers, parents and school teachers who will ensure that children adhere to healthy eating and appropriate lifestyle practices.

\section{Acknowledgements}

We acknowledge the funding support given by the Office of Research Innovation and Development, University of Ghana. The requisite support and co-operation given by study participants and their parents/guardians, school heads and their staff and all who helped with the data collection, is greatly appreciated.

MSA conceived the study and contributed to its design. She was also responsible for acquisition of funding and provided general supervision of the research. PA and AKA were responsible for the design, acquisition of the logistics for the study. PA carried out data collection, analysis and drafting of the manuscript. EA, OAH and ROB contributed in drafting the manuscript and revising it critically for important intellectual content.

\section{Competing Interests Statement}

The authors declare that there are no competing or potential conflicts of interest.

\section{References}

Alhassan, A., \& Alhassan, F. (2014). An assessment of the operational challenges of the Ghana School Feeding Programme. International Journal of Business, Management and Social Research, 2(8), 154.

Atta, G. P., \& Manu, J. (2015). Ghana School Feeding Program: A Retrospective Review. International Journal of Innovative Research and Development, 4(8), 1-9.

Bowman, B. A. B., \& Russell, R. M. (2001). Present knowledge in nutrition. Pan American Health Org.

Contento, I., Balch, G. I., Bronner, Y. L., Lytle, L., Maloney, S., Olson, C., \& Swadener, S. S. (1995). The effectiveness of nutrition education and implications for nutrition education policy, programs, and research: a review of research. Journal of Nutrition Education and Behaviour, 3(4), 2-34. 
Eliakim, A., Kaven, G., Berger, I., Friedland, O., Wolach, B., \&Nemet, D. (2002). The effect of a combined intervention on body mass index and fitness in obese children and adolescents-a clinical experience. European Journal of Pediatrics, 161(8), 449-454. https://doi.org/10.1007/s00431-002-0980-2

Global School-based Health Survey (GSHS). (2008). Retrieved from http://www.who.int/chp/gshs/2008_Ghana_GSHS_Country_Report.pdf.

Hu, C., Ye, D., Li, Y., Huang, Y., Li, L., Gao, Y., \& Wang, S. (2010). Evaluation of a kindergarten-based nutrition education intervention for pre-school children in China. Public Health Nutrition, 13(02), 253-260. https://doi.org/10.1017/S1368980009990814

Kaiser, L. L., \& Townsend, M. S. (2005). Food insecurity among US children: Implications for nutrition and health. Topics in Clinical Nutrition, 20(4), 313-320. https://doi.org/10.1097/00008486-200510000-00004

Lobstein, T., Baur, L., \& Uauy, R. (2004). Obesity in children and young people: a crisis in public health. Obesity Reviews, 5(s1), 4-85. https://doi.org/10.1111/j.1467-789X.2004.00133.x

Regenerative Health and Nutrition Programme. (2007). Dangers of Physical Inactivity. Retrieved October 8, 2011, from http://www.rhnp.org/new/rhnp34/index1.php?id=0002\&pgtid=3\&cntid=hl\&pgt=Healthy\%20Lifestyle

Shariff, Z. M., Bukhari, S. S., Othman, N., Hashim, N., Ismail, M., Jamil, Z., . . Hussein, Z. A. M. (2008). Keywords Adolescents Children Chinese College Students Health Health Education Health Promotion Internet Knowledge Online Learning Physical Activity Smoking adolescents education global health healthhealth behavior health education health promotion physical activity technology.

Sherman, J., \& Muehlhoff, E. (2007). Developing a nutrition and health education program for primary schools in Zambia. Journal of Nutrition Education Behaviour, 39(6), 335-342. https://doi.org/10.1016/j.jneb.2007.07.011

SitiSabariah, B., Zalilah, M., Norlijah, O., Normah, H., Maznah, I., Laily, P., . . MH, Z. A. (2006). Reliability and validity of the instrument used in the HELIC (Healthy Lifestyle in Children) study of primary school children's nutrition knowledge, attitude and practice. Malaysian Journal of Nutrition, 12(1), 33-44.

Steiner-Asiedu, M., Addo, P., Bediako-Amoa, B., Fiadjoe, F. Y., \& Anderson, A. K. (2012). Lifestyle and nutrition profile of overweight and obese school children in the Ga-East District of Ghana. Asian Journal of Medical Sciences, 4(3), 99-104.

Williams, D. P., Going, S. B., Lohman, T. G., Harsha, D. W., Srinivasan, S. R., Webber, L. S., \& Berenson, G. S. (1992). Body fatness and risk for elevated blood pressure, total cholesterol, and serum lipoprotein ratios in children and adolescents. American Journal of Public Health, 82(3), 358-363. https://doi.org/10.2105/AJPH.82.3.358

World Health Organisation. (2007). Sex and age specific BMI curves. Retrieved October 25, 2011, from http://www.who.int/growthref/who2007_bmi_for_age/en/index.html

\section{Copyrights}

Copyright for this article is retained by the author(s), with first publication rights granted to the journal.

This is an open-access article distributed under the terms and conditions of the Creative Commons Attribution license (http://creativecommons.org/licenses/by/4.0/). 